\begin{tabular}{l} 
Sharif University of Technology \\
Scientia Iranica \\
SCIENTIA \\
IRAN \\
Thanstions A: Civil Engineering \\
\hline
\end{tabular}

\title{
Behavioral analysis of vehicle-pedestrian interactions in Iran
}

\author{
A. Sheykhfard and F. Haghighi* \\ Department of Civil Engineering, Babol Noshirvani University of Technology, Babol, Iran.
}

Received 7 June 2016; received in revised form 4 October 2016; accepted 6 March 2017

\section{KEYWORDS \\ Pedestrian accidents; \\ Vehicle-pedestrian \\ collision; \\ Vehicle driver \\ behavior; \\ Pedestrian behavior; \\ DREAM method.}

\begin{abstract}
Statistics for the injured and killed pedestrian accidents in recent years have expressed high vulnerability of this group of road users. By identifying influential factors in the interactions of pedestrians-vehicles and representing appropriate solutions to reduce the impact of these factors, the possibility of such interactions and, consequently, the relative accidents can be reduced. In the present research, based on Naturalistic Driving Studies (NDS), the driving behaviors of 29 drivers of vehicles were investigated. In the local urban routes of Babol City in Mazandaran, Iran, 289 vehicle-pedestrian interactions at the traffic peak hours were determined. By analyzing the interactions using the DREAM method (Driving Reliability and Error Analysis Method), the risk factors were identified and two causal patterns were determined for pedestrian crossings and places lacking any pedestrian crossings. Drivers talking to passengers and listening to music were among the factors influencing the occurrence of interactions at pedestrian crossings. Unexpected behaviors by pedestrians while crossing (such as sudden running, crossing careless of the traffic flow, and crossing without obtaining the permission from the vehicle driver) played a substantial role in the occurrence of interactions in places without pedestrian crossings. Finally, some solutions were proposed for reducing the chances of occurrence of interactions.
\end{abstract}

(C) 2018 Sharif University of Technology. All rights reserved.

\section{Introduction}

The traffic accident is a major cause of death in human societies. According to the World Health Organization, about 1.2 million people are killed due to traffic accidents in which pedestrians have a considerable share of these casualties [1]. In Iran, according to a tenyear report of the Iranian Legal Medicine Organization, about 235 thousand people were killed on the road, and pedestrians constitute 23.2 percent of the casualties.

*. Corresponding author.

E-mail addresses: A.sheykhfard@nit.ac.ir (A. Sheykhfard)

Haghighi@nit.ac.ir (F.Haghighi).

doi: $10.24200 /$ sci.2017.4201
An interaction is an observable situation in which two or more road users approach each other in space and time to such an extent that there is a risk of collision if their movements remain unchanged (Figure 1).

By identifying the factors involved in the occurrence of vehicles-pedestrian interactions, it is possible to reduce interactions and, consequently, decrease the number and severity of pedestrian accidents by developing instructions and conducting traffic control operations. This research makes attempts to examine the factors influencing the occurrence of interactions between vehicles and pedestrians. To fulfill this purpose, based on naturalistic driving studies, 29 participants were examined, and after assessing the influencing factors, causal patterns of occurrence of interactions were identified. 


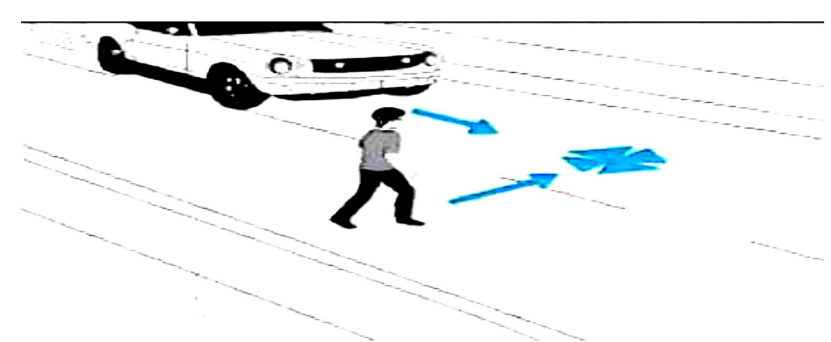

Figure 1. Schematic view of a traffic interaction.

\section{Background}

Accident patterns are studied through statistical analyses of accidents, and accident databases are normally used as a reference for these studies [2]. Reports available in accident databases include information on the wounded and killed victims of traffic accidents. These databases, therefore, do not offer much information on pre-accident events and specifications of the accidents occurred [3,4]. Hence, more attention has been paid recently to naturalistic driving studies on factors involved in accident occurrence and causal patterns of accidents. In driving behavior studies, vehicles are equipped with cameras and sensors that allow for filming and recording information on people's driving and environmental condition of route traffic flow before and after interactions, semi-accidents, and traffic accidents [5]. Hunter et al. [6] studied 975 interactions between pedestrians and vehicles. Influential factors in the occurrence of interactions included analysis by logistic regression appointed that short distance to crosswalks, low speed of vehicle, pedestrians at the curb of the crosswalks, yielding by vehicle driver of the opposite direction, group of pedestrians and students. Sucha (2014) examined the factors affecting the behavior of drivers and pedestrians by analyzing 1584 interactions between vehicle and pedestrian using the Logistic Regression model. Important factors such as vehicle speed, headway and gap spacing between vehicles, and traffic flow density affected the behavior of pedestrians to cross; the use of mobile phone by drivers, short distance to pedestrians, and low-density traffic flow reduced drivers' performance in interactions with pedestrians [7]. Seipone (2013) studied distracting behaviors of drivers and pedestrians in vehiclepedestrian interactions. Results of the study based on mathematical probability indicated low performance of the majority of drivers (80.4\%) and pedestrians. Using a mobile phone, texting, and talking to passengers were considered the most serious distracting behaviors that caused the non-performance of the drivers; in addition, talking with other pedestrians and the use of mobile phones by pedestrians were considered the distracting behaviors on pedestrians in interactions [8]. Factors such as age, gender, group of pedestrians and pedestrian crossing styles (walking or running during crossing) were the main causes of 594 interactions based on the study of Langbroek et al. (2012) [9].

The DREAM method (Driving Reliability and Error Analysis Method) was used successfully in European pedestrian accidents safety projects for the first time [10]. To assess the validity of the DREAM in analyzing the causes of accidents in different scenarios, Warner and Sandin (2010), in collaboration with several researchers, focused on the causes of accidents, and demonstrated that the DREAM is considerably useful (about $80 \%$ ) for identifying the causes of accidents and determining causal patterns of accidents [11]. Habibovic et al. (2013) used the DREAM method to study the factors influencing the interactions between vehicles and pedestrians. They studied the behavior of 45 drivers during 95 vehicle-pedestrian interactions and determined two causal patterns for the occurrence of interactions [12].

\section{Methodology}

\subsection{Dream method}

By connecting different factors with mutual interactions, the DREAM leads to the identification of the major causes of accidents and traffic interactions. In its tree diagram, the DREAM method consists of predefined links, showing the cause of an accident based on the relationship between influencing factors. The predefined diagrams of the DREAM allow for classification of different diagrams of causes of accidents, which could be used as the basis for identifying common patterns of accidents and interactions [13]. The DREAM consists of three main elements: accidents model, classification plan, and analysis process.

A. The accident model: According to this section, accidents occur due to malfunctions by the following three agents: human, technology, and organization. The human agent includes the driver's performance including observation, interpretation, and planning in the course of timely decision-making while driving in the environment. Any mistake or delay in decision-making and the performance leads to accidents. Occurrence of accidents due to any defect or lack of proper vehicle productivity (such as technical flaws) and traffic flow problems (e.g. driver's limited vision, road lighting system defect, etc.) is associated with technology. The organization includes all of the appropriate institutions for route construction and maintenance, status of boards and traffic control systems, vehicle design, and road design where malfunction of this component increases the probability of accidents;

B. The classification scheme: DREAM diagrams are composed of two parts: the phenotype and genotype. Phenotypes are vital events shown by time, 
Table 1. Phenotypes and specific phenotypes of DREAM.

\begin{tabular}{ll}
\hline Phenotypes & \multicolumn{1}{c}{ Specific phenotypes } \\
\hline Timing & Too early action; too late action; no action \\
Speed & Too high speed; too low speed \\
Distance & Too short distance \\
Direction & Wrong direction \\
Force & Surplus force; insufficient force \\
Object & Adjacent object \\
\hline
\end{tabular}

distance, speed, and force dimensions. Therefore, any malfunction on the side of the driver in the process of adapting to the natural traffic flow of the environment occurs before the accident (for example, consider high vehicle speed, which causes accident due to the driver's lack of control in adapting to the traffic flow of the environment). Genotypes are various factors that lead to the driver's malfunction as a phenotype and, ultimately, lead to accidents due to a series of flaws caused by the three factors mentioned before. The DREAM manual presents detailed descriptions of classifications and definitions of genotypes and phenotypes of this method along with examples. If adequate information on genotypes and phenotypes is available, they can be studied in a specific state [14].

The phenotype for an accident can be identified based on the DREAM method, as shown in Table 1 . In other words, the DREAM diagram of each accident finally is linked to a specific phenotype. Lack of precise timing of functions, inappropriate control of vehicle speed, failure to respect the safety distance, driving the vehicle in the wrong direction, failure to use adequate forces at the time of using the pedal systems, and lack of adequate driving control are among the phenotypes, which cause accidents with the malfunction of drivers. Similar to phenotypes, genotypes are divided into the groups of general and particular genotypes. Particular genotypes are the cause of the emergence of general genotypes, and one general genotype can cause another general genotype. Accordingly, a DREAM diagram includes one phenotype and several genotypes connected to each other in a series. These connections show the reason for the occurrence of an accident or interaction. By classifying various DREAM diagrams, it is possible to identify the causes of accidents and interactions and use the results to determine the pattern of such accidents. DREAM genotypes are classified into the following three groups in the manual of this method: human factor, technology factor, and organization (Table 2);

C. The method: The connection of phenotypes and genotypes at the time of analyzing the cause of an accident is established based on several principles, which are called the stop rules. In analyzing an accident or interaction, several factors (genotypes) may lead to the emergence of a phenotype. The relationship between these factors and their ultimate relationship with the phenotype (the last phase of analysis) is established using the three principles of the stop rules which are as follows:

(a) Specific genotypes have the status of terminal events. Therefore, if a specific genotype is most likely the cause of a general consequent, then specific genotype is chosen and the analysis stops;

(b) If there exist no general or specific genotypes linked to the chosen consequent, the analysis stops;

(c) If none of the available specific or general genotypes for the chosen consequent is relevant, given the information available about the accident, the analysis stops.

In view of the above principles, analysis of an accident takes place in reverse. In other words, the DREAM diagram starts with the observed phenotype and the general and particular genotypes, which are the cause of one another and the phenotype, leading to smaller constituting agents using the above principles. To adapt the ultimate project objective to the method's objective, it is possible to make modifications to the analysis component of the mentioned method (based on the project's data) to increase precision of the results obtained from this method [12]. In this research, to compare the information obtained from watching the recorded films with the factors defined in the DREAM, some particular genotypes (such as driver's listening to music, driver's talking to passengers, improper road crossing by pedestrians, and crossing of pedestrians without permission) are defined, which will contribute to the identification of the general genotypes of the method's diagrams.

\subsection{Study site}

To identify the factors involved in occurrence of vehiclepedestrian interactions and determine causal patterns of the interactions occurred, the naturalistic driving studies of 29 vehicle drivers were examined. In this research, the participants travelled the four-kilometer high-traffic urban road of Babol City in Mazandaran Province (straight route with a length of 4 kilometers between Oghaf to Hamzehkola Square, and vice versa) at peak hours with maximum Annual Average Daily Traffic (AADT) of 4,000 veh/day and $30 \mathrm{k} / \mathrm{hr}$ posted speed (Figure 2). The rout has two lanes (each band) and undivided with high numbers of pedestrians crossing the street due to the existence of two schools, a university, and numerous shopping centers. 
Table 2. Main causes of accidents (genotypes).

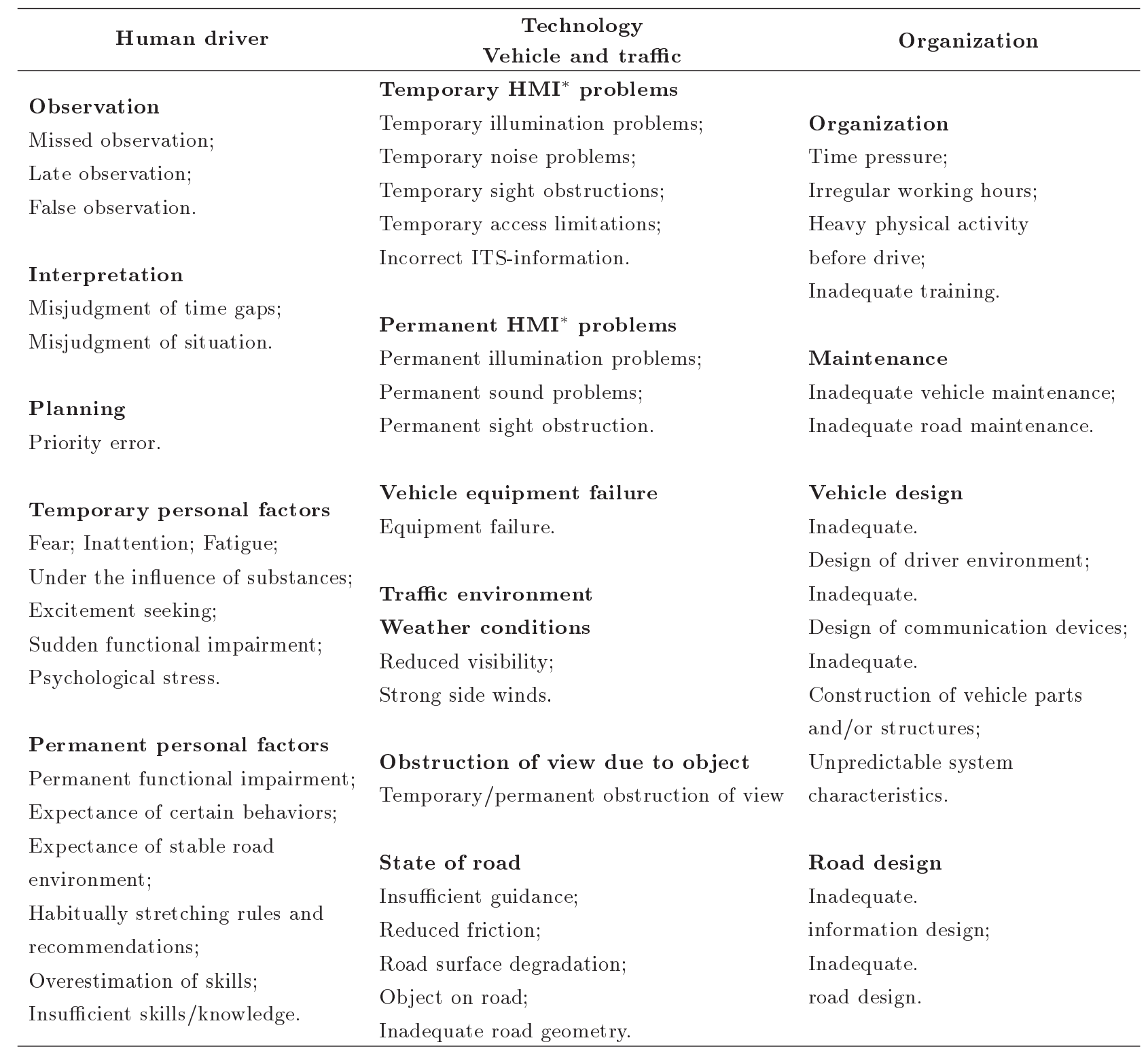

*HMI: Human-Machine-Interface.

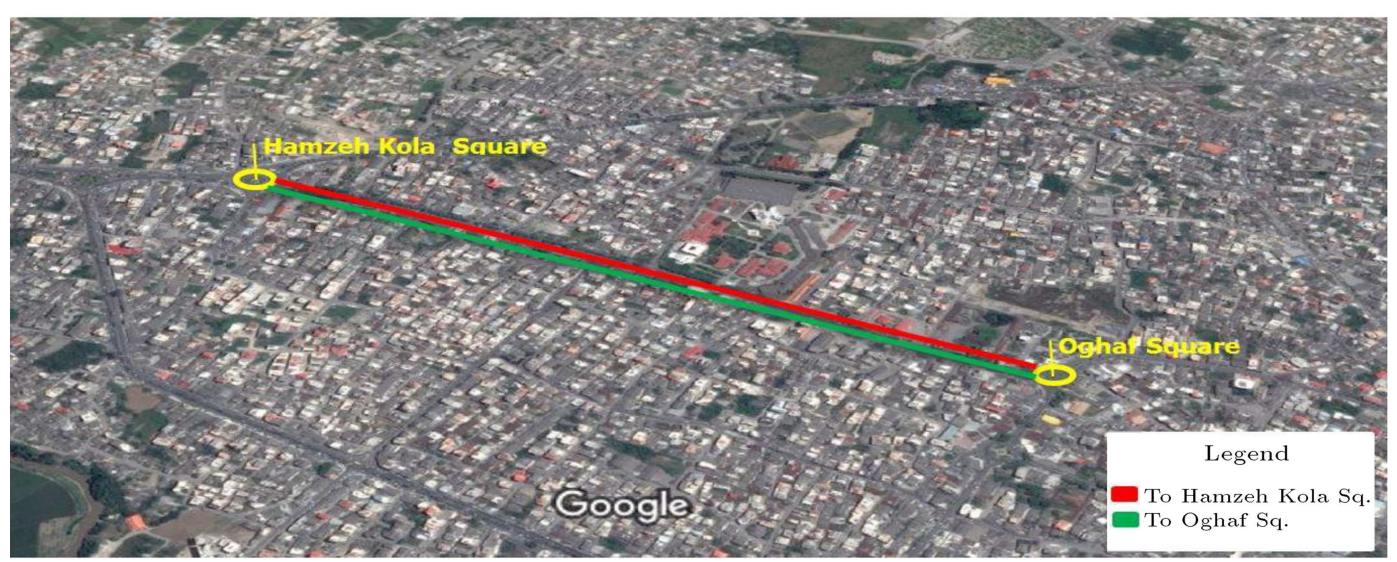

Figure 2. Selected route with a length of 4 kilometers between Oghaf to Hamzehkola Square and vice versa. 


\subsection{Data collection}

A total of 29 participants (22 males and 7 females) were involved in the study (ranging from 18 to 45 years old) with different jobs and levels of education. All participants had a valid driving license with average 6.6 years of driving (ranging from 2 to 14 years) and average of $5200 \mathrm{~km}$ per year (ranging from 3000 to $10,000 \mathrm{~km})$. Data collection was conducted in two days (on April 18th and 21th, 2015) at peak hours (7:30-8:30, 12:30-13:30, and 17:30-18:30). Driving performances of drivers were recorded via three cameras installed in the vehicle (Figure 3 ). The first camera was an HD dual camera $(640 * 480$ pixels and 25 frame/sec) installed below each vehicle's front mirror to film the inside of the car and outside of the vehicle (the road ahead) simultaneously. The other HD camera (720*480 pixels and 30 frame/sec) was installed on the passenger seats to film the vehicle's pedals. In this research, 289 vehicle-pedestrian interactions occurred, and accidents could have occurred without a timely, proper performance of drivers or pedestrians.

Figure 4 shows an example of the DREAM chart that reveals the causal patterns for the car driver involved in a car-to-pedestrian interaction. This DREAM chart is read from right to left, reversing the causal sequence, and can be interpreted in the following way.

According to Figure 4, driver talking to passengers (specific genotype) distracts drivers (general genotype) from the road and the driver cannot see the conflict pedestrian at some key moments prior to the interaction. As a result of this lack of attention, drivers fail to perfectly judge the environmental condition (general genotype) as a result of late observation (general genotypes) of the present pedestrians. Consequently, due to the high speed of the vehicle and driver's late reaction (specific phenotypes), the vehicle collides with pedestrians.

\section{Results}

\subsection{Dream diagrams}

In analyzing the collision between pedestrians and vehicles, the interactions between several pedestrians and vehicles, which were trying to cross the street simultaneously, were considered a single sample. After examining the films recorded by the cameras inside the vehicles, it was found that pedestrians and drivers of vehicles interact in four different states (passing from left/right side of the street at pedestrian cross-
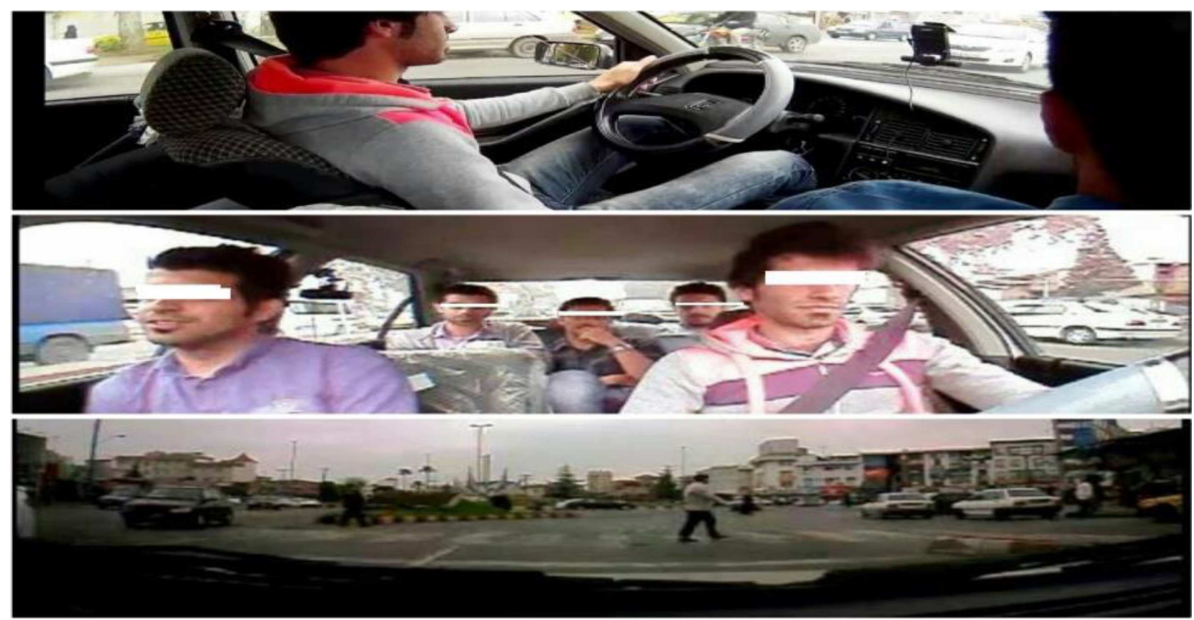

Figure 3. View of recording via the three cameras installed in the vehicle.

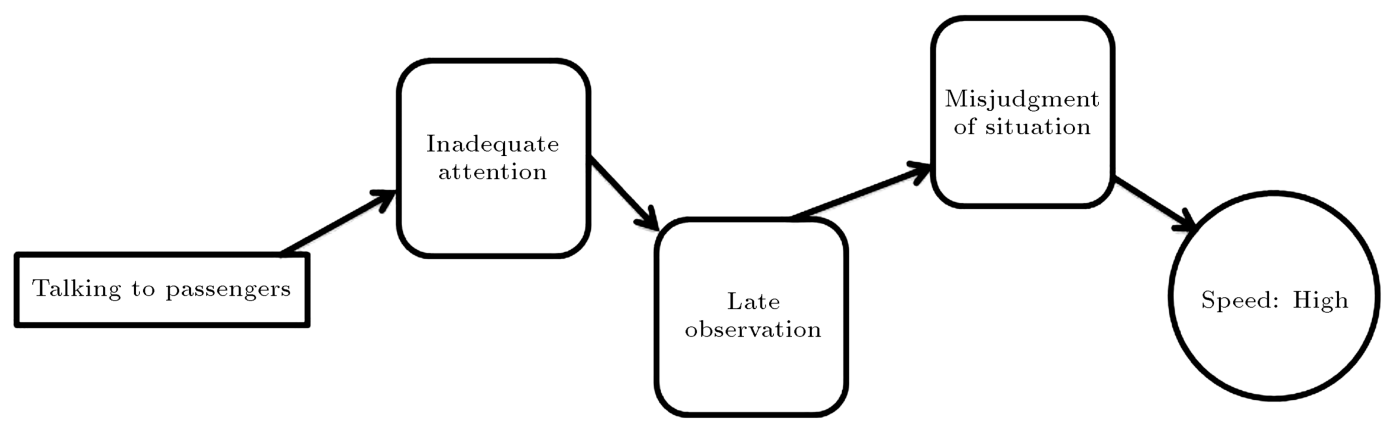

Figure 4. Example of an individual causation chart for a driver involved in a vehicle-pedestrian incident. The chart should be read from right to left, reversing the causal chain. 


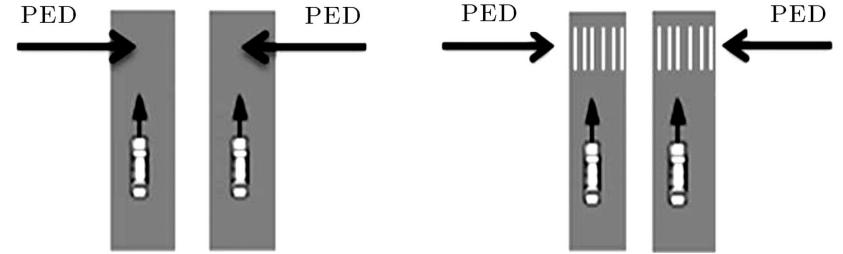

Figure 5. Two groups describing geometries of the vehicle and pedestrian paths immediately prior to the interaction.

ing or places without pedestrian crossings). Of the 289 vehicle-pedestrian interactions observed in driver's behavioral studies, 93 interactions occurred at pedestrian crossings and 196 interactions occurred in places lacking pedestrian crossings. To determine precisely the factors involved in occurrence of interactions and to develop a causal pattern for their occurrence, the interactions were investigated and studied in the two following groups: interactions at pedestrian crossings; interactions at places without pedestrian crossings (Figure 5).

\subsubsection{Dream diagram for interaction at pedestrian crossings}

A total of 93 interactions at different pedestrian crossings along the study route were identified by the cameras installed inside vehicles. Afterwards, using the DREAM manual and the mentioned process, first, the phenotypes creating the interactions and, then, the general and particular genotypes involved in series and tree-like structures in the occurrence of the phenotype were identified. Moreover, 93 separate diagrams were prepared for all interactions, and then the influencing factors in the diagrams were merged and diagrams were classified. Figure 6 shows the dream diagram of overall results of analyzing the causes of interactions at pedestrian crossings based on their causal patterns. It should be noted that the figure inside the parentheses next to each genotype and phenotype of causal patterns shows their frequency at the time of occurrence of interactions.

\subsubsection{Dream diagram for interactions at places without crossings}

The classified DREAM diagram for 196 interactions at places without pedestrian crossings is shown in Figure 7.

\subsection{Interpretation of dream diagrams}

According to Figure 6, drivers talking to passengers and listening to music (particular genotypes) are among the influencing factors, leading to the lack of attention in drivers (general genotype) by distracting them from the road. As a result of this lack of attention, drivers fail to perfectly judge the environmental condition (general genotype) as a result of late observation or lack of observation (general genotypes) of present pedestrians. Consequently, due to the high speed of the vehicle and late reaction (particular

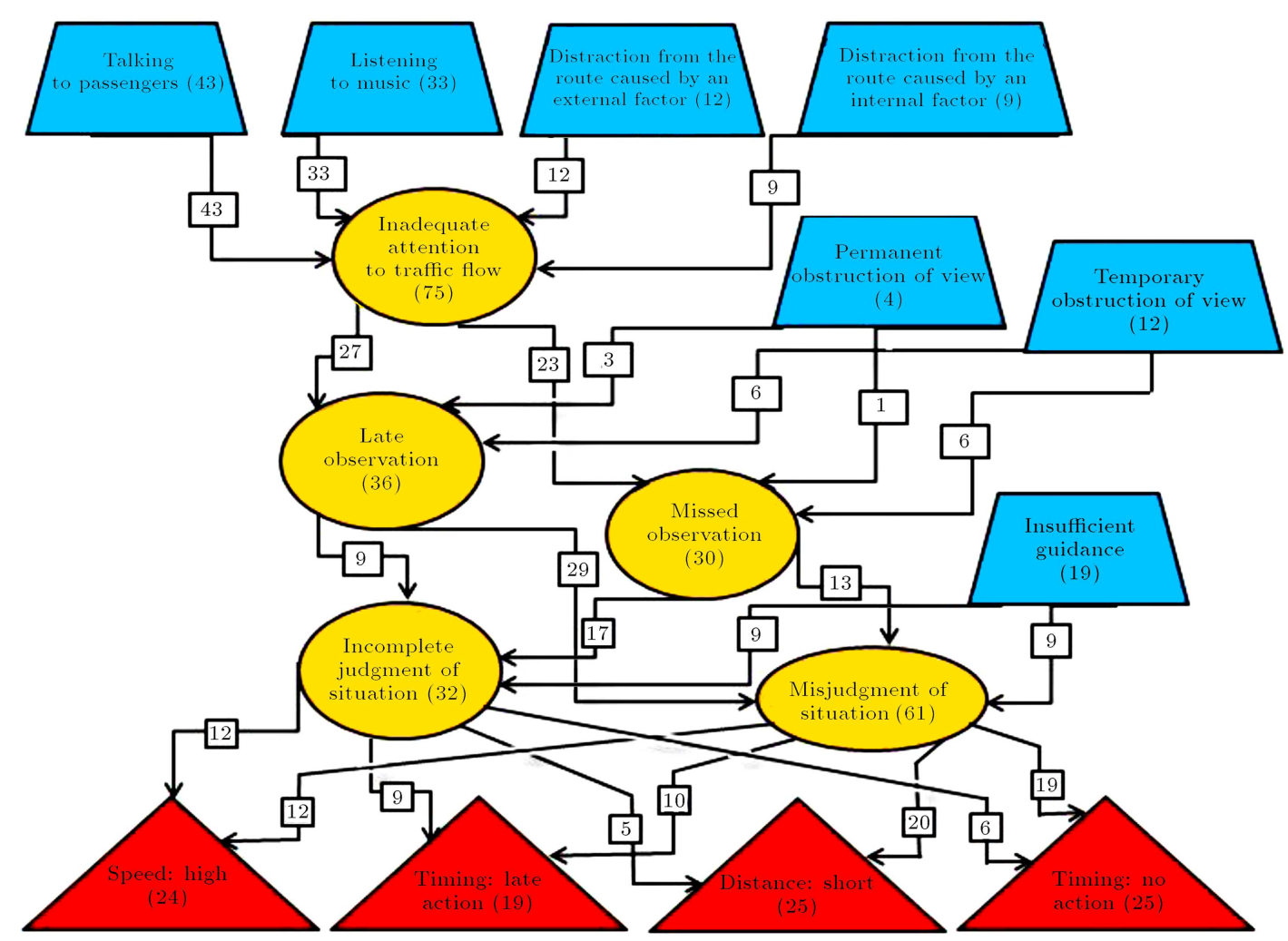

Figure 6. Causal patterns of 93 vehicle-pedestrian interactions at crossings. 


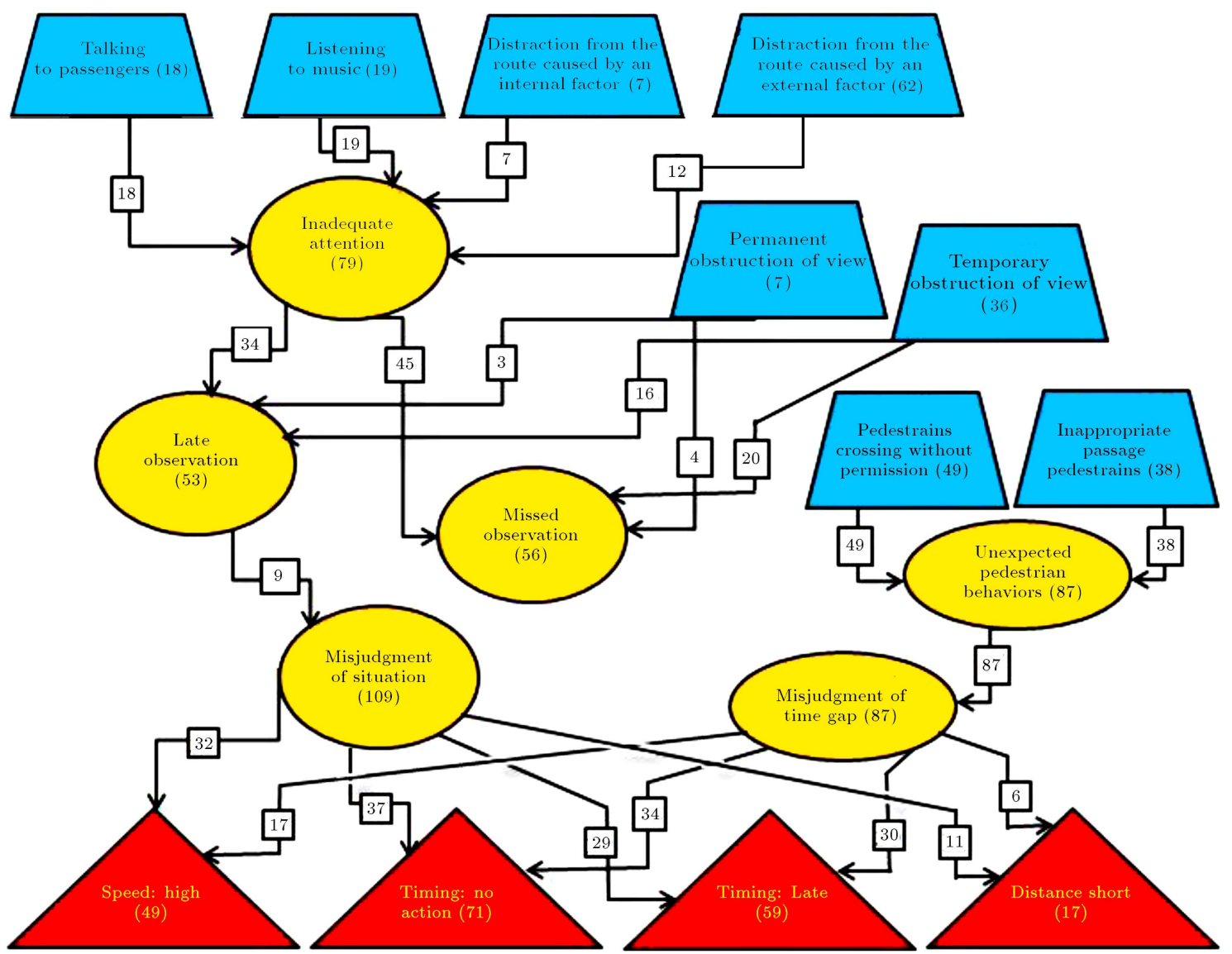

Figure 7. Causal patterns of 196 vehicle-pedestrian interactions at places without crossing.

phenotypes), the vehicle interacts with pedestrians. Due to the lack of control equipment and road guides (general genotype), such as warning signs for pedestrian crossings (particular genotype), drivers made mistakes in making proper decisions and judgments (general genotype) and failed to prevent interactions at the right time by reducing the speed and valuing the safety distance between their vehicle and pedestrian crossings (particular phenotypes).

As seen in Figure 7, at many interactions, the driver fails to pay adequate attention (general genotype) by being distracted from the outside environment such as stores, etc. (particular genotype). Due to the same factor along with temporary limited vision (particular genotype), such as the presence of other parked vehicles or advertisement boards along the road, drivers fail to make accurate judgment on the environment (general genotype) at the time of facing pedestrians because of late observation and lack of observation (general genotypes). As a result, due to the lack of speed reduction and demonstration of a proper and timely reaction (particular phenotypes), an interaction takes places between the pedestrians and vehicle. On the other hand, risky behavior patterns of pedestrians passing the crossings, such as suddenly running across the street, crossing the street without permission of approaching vehicles, and crossing the street careless of the previous route traffic and the current traffic (particular genotypes), are among the behaviors (general genotypes) which cannot be predicted by drivers of vehicles (general genotype). As a result of these factors, drivers fail to have correct judgment of the time suitable for safe passage of pedestrians (general genotype). Consequently, a late reaction and short distance to pedestrians make drivers fail to show a timely reaction at the time of driving.

Research results indicated that, at pedestrian crossings, factors, such as driver's listening to music and talking to passengers, cause interactions due to their negative effect on performance. This result complies with the finding by Emre and Engin [15]. To explain this result, it could be stated that such driving behaviors distract drivers from the route conditions and increase the risk of interactions and vehicle-pedestrian accidents. Analysis of the DREAM diagrams of interactions at places without crossings indicated that driver's failure in accurately predicting the time interval for safe passage of the pedestrians, caused by unexpected behaviors of pedestrians (such as crossing along the street, failure to obtain permission of drivers before passing, and lack of attention to the past traffic flow and the traffic flow at the time of crossing 
Sites without pedestrian crossing

Sites with pedestrian crossing

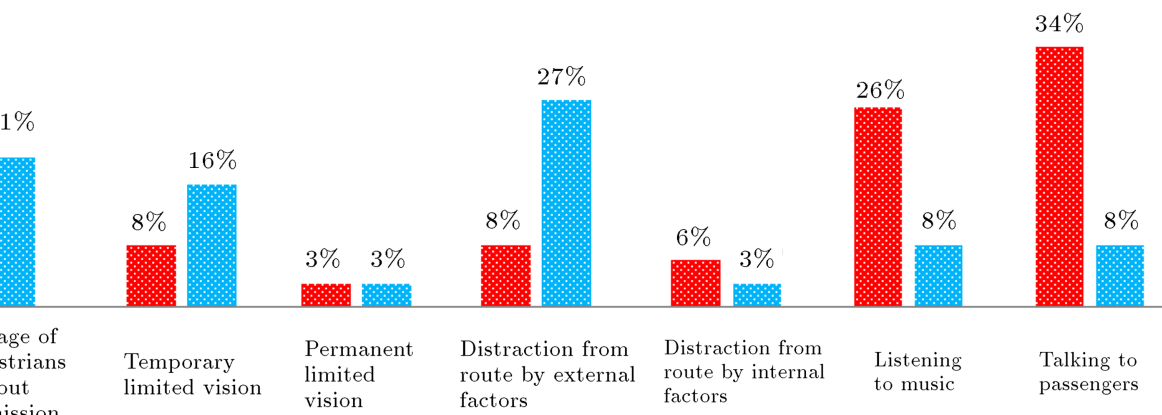

Figure 8. Causes of vehicle-passenger interactions.

the street), is among the main causes of interactions. In this research, pedestrians asked for permission to cross the stress by their hand movements that enabled drivers to show a better performance. This factor reduced the probability of interactions similar to the research by $\mathrm{Wu}$ and Xiangling [16]. In the present study, pedestrians' lack of attention to the traffic flow at the time of crossing the street increased the probability of interactions. In addition, Lenard and Hill also introduced this factor as a factor involved in occurrence of vehicle-pedestrian interactions [17]. Another result of this research was pedestrians' lack of attention for the road, which increased the chances of interactions. According to Neale et al. [18], this factor is among the main causes of occurrence of interactions between vehicles and pedestrians (Figure 8).

\section{Discussion and conclusion}

By identifying the influencing factors and determining the causal patterns of occurrence of vehicle-pedestrian interactions, it is possible to develop different plans and actions to reduce casualties and mortalities in pedestrians and increase their safety in traffic accidents. Due to the possibility of occurrence of errors at the time of recording and deriving information from accidents databases and due to the lack of inclusive studies on causes of accidents, Naturalistic Driving Studies (NDS) have attracted more attention in recent years. In naturalistic driving studies, it is possible to investigate all available information on the period before and during accidents or interactions via the cameras and sensors installed inside vehicles. Several methods are employed by researchers to determine causal patterns of occurrence of accidents and traffic interactions in traffic safety studies. The DREAM method is among the most common and useful methods for this purpose due to its high information processing speed and precision. In the present study, through camera-aided investigations, the behaviors of 29 drivers were studied during 280 vehicle-pedestrian interactions on a local urban route in Babol City in Mazandaran
Province. By inspecting the recorded films and using DREAM, two causal patterns of interactions at places with and without pedestrian crossings were developed, and factors involved in occurrence of the interactions were also identified. At pedestrian crossings, factors, such as driver's listening to music, driver's talking to passengers, and lack of warning signs at sites allocated to pedestrians, were the causes of vehicle-pedestrian interactions on the study route. At places lacking pedestrian crossings, unexpected behaviors shown by pedestrians crossing the street and drivers' distraction from the route increased the chances of interactions. Sudden running, crossing without permission, and crossing the street without looking at the route were among the unexpected pedestrian behaviors, which led to malfunction of drivers.

In view of the research results, installation of warning signs at pedestrian crossings, installation of boards and advertisement systems to prevent drivers from talking to passengers and paying inadequate attention to driving, and construction of pedestrian passageways in high-traffic places lacking crossings along the study route are among the solutions that could reduce the probability of vehicle-pedestrian interactions and increase safety of pedestrians.

\section{References}

1. WHO, Global Status Report on Road Safety: Time for Action, World Health Organization (WHO), Geneva, Switzerland (2009).

2. OECD Staff, Road Accidents: On-site Investigations, Organization for Economic Cooperation and Development (OECD), Paris (1988).

3. Larsen, L. "Methods of multidisciplinary in-depth analyses of road traffic accidents", Journal of Hazardous Materials, 111(1-3), pp. 115-122 (2004).

4. Shinar, D., Treat, J.R., and McDonald, S.T. "The validity of police reported accident data", Accident Analysis and Prevention, 15(3), pp. 175-191 (1983).

5. Dingus, T.A., Klauer, S.G., Neale, V.L., Petersen, A., Lee, S.E., and Sudweeks, J., The 100-car naturalistic 
driving study. Phase II: Results of the 100-car field experiment, Virginia Tech Transportation Institute, National Highway Traffic Safety Admin (NHTSA), p. 422 (2006).

6. Hunter, E., Salamati, K., Elefteriadou, L., Sisiopiku, V., Rouphail, N., Phillips, B., and Schroeder, B. "Driver yielding at unsignalized midblock crossings", Proceedings of the 94th Transportation Research Board Annual Meeting, Washington, D.C. (2015).

7. Sucha, M. "Pedestrians and drivers: their encounters at zebra crossings", 8th International Traffic Expert Congress, 8 to 9 May, Warsaw (2014).

8. Seipone, B. and Mphele, M. "Who owns the road? Exploring driver and pedestrian behavior at zebra/pedestrian crossings in gaborone", Botswana. British Journal of Arts and Social Sciences, 1(13), pp. 2046-9578 (2013).

9. Langbroek, J., Ceunynck, T., Daniels, S., Svensson, A., Laureshyn, A., Brijs, T., and Wets, G. "Analyzing interactions between pedestrians and motor vehicles at two-phase signalized intersections-an explorative study combining traffic behavior and traffic conflict observations in a cross-national context", International Cooperation on Theories and Concepts in Traffic Safety(ICTCT), Proceedings (CD-Rom) p. 1-21 (2012).

10. Habibovic, A. and Davidsson, J. "Requirments of a system to reduce car-to-vulnerable road user crashes in urban intersections", Accident Analysis and Prevention, 43(4), pp. 1570-1580 (2011).

11. Warner, H.W. and Sandin, J. "The intercoder agreement when using the driving reliability and error analysis method in road traffic accident investigations", Accident Analysis and Prevention, 48(5), pp. 527-536 (2010).

12. Habibovic, A., Tivesten, E., Uchida, N., Bargman, J., and Ljung, M. "Driver behavior in car-to-pedestrian incidents: An application of the driving reliability and error analysis method (DREAM). Accident Analysis and Prevention, 50, pp. 554-565 (2013).

13. Sandin, J. "An analysis of common patterns in aggregated causation charts from intersection crashes", Accident Analysis and Prevention, 41, pp. 624-632 (2009).

14. Warner, H.W., Ljung, M., Sandin, J., Johansson, E., and Björklund, G. "Manual for DREAM 3.0, Driving
Reliability and Error Analysis Method", Chalmers University of Technology, Gothenburg, Sweden (2008).

15. Emre, O. and Engin, E. "Driver status identification from driving behavior signals", Digital Signal Processing For In-Vehicle Systems and Safety, pp. 31-55 (2012).

16. Wu, C. and Xiangling, Z. "Pedestrian gestures increase driver yielding at uncontrolled mid-block road crossings", Accident Analysis and Prevention, 70, pp. 235244 (2014).

17. Lenard, J. and Hill, J. "Interaction of road environment, vehicle and human factors in the causation of pedestrian accidents," in Proceedings of International Conference on ESAR (Expert Symposium on Accident Research), 3-4 September , Hannover, Germany, (2004).

18. Neale, V., Dingus, T., Klauer, S.H., Sudweeks, J., and Goodman, M. An Overview of the 100-Car Naturalistic Study and Findings, Virginia Tech Transportation Institute, National Highway Traffic Safety Administration, United States, pp. 05-0400 (2011).

\section{Biographies}

Abbas Sheykhfard received his BSc degree in Civil Engineering from Jundi Shapur University in 2013. He was accepted for MSc degree in Road and Transportation Engineering in Civil Engineering Department at Babol Noshirvani University of Technology in 2013. He carried out his research thesis titled "Modeling driver's behavior in encountering pedestrians" under supervision of Dr. Haghighi. His research interests are traffic calming and road user behavioral analysis.

Farshidreza Haghighi received the BEng degree in Civil Engineering from the Babol Noshirvani University of Technology (NIT) in 2001 and the $\mathrm{PhD}$ degree in Transportation Engineering from the Iran University of Science and Technology, in 2011.

$\mathrm{He}$ is currently an Assistant Professor at the Department of Civil Engineering, Babol Noshirvani University of Technology (NIT). His research interests lie in the general areas of traffic safety, traffic calming, and urban transportation planning. 